\title{
A flexible anisotropic self-powered piezoelectric direction sensor based on double sided $\mathrm{ZnO}$ nanowires configuration
}

\author{
Eiman Satti Osman, Chan Oeurn Chey, Magnus Willander and Omer Nur
}

\author{
Linköping University Post Print
}

Tweet

N.B.: When citing this work, cite the original article.

Original Publication:

Eiman Satti Osman, Chan Oeurn Chey, Magnus Willander and Omer Nur, A flexible anisotropic self-powered piezoelectric direction sensor based on double sided $\mathrm{ZnO}$ nanowires configuration, 2015, Nanotechnology, (26), 9, 095502.

http://dx.doi.org/10.1088/0957-4484/26/9/095502

Copyright: IOP Publishing: Hybrid Open Access

http://www.iop.org/

Postprint available at: Linköping University Electronic Press

http://urn.kb.se/resolve?urn=urn:nbn:se:liu:diva-115311 


\title{
A flexible anisotropic self-powered piezoelectric direction sensor based on double sided $\mathrm{ZnO}$ nanowires configuration
}

\author{
E. S. Nour*, C. O. Chey, M. Willander, and O. Nur
}

Department of Science and Technology (ITN), Linköping University, Campus Norrkoping, SE-601 74 Norrkoping, Sweden.

\begin{abstract}
We have successfully synthesized highly dense and well aligned zinc oxide $(\mathrm{ZnO})$ nanowires (NWs) on the two sides of a PEDOT: PSS substrate by a single step low temperature hydrothermal method. The grown sample was used to fabricate a double sided piezoelectric nanogenerator (NG). The maximum harvested output power density from the fabricated double sided NG configuration was about $4.44 \mathrm{~mW} / \mathrm{cm}^{2}$. The results obtained from the present double sided NG were approximately double the output from a single side. In addition to that, the voltage polarity of the harvested voltage from the two sides of the NG has been investigated. The results showed that upon bending, an anisotropic voltage polarity is generated on the two sides. Indicating that, this double sided NG can be used as a self-powered voltage polarity based direction sensor. The results of the present flexible double sided NG is very promising for harvesting energy from irregular mechanical energy sources in the surrounding environment. In addition, the fabricated configuration showed stability for sensing and can be used in surveillance and security applications.
\end{abstract}

Keywords: Hydrothermal growth, Nanogenerator, Energy harvesting, Piezoelectric nanowires.

* Corresponding author E-mail: eiman.satti.osman@liu.se 


\section{Introduction}

Since the past decade harvesting energy has attracted increasing attention. This increased interest is due to the potential applications in developing self-powered systems based on nanostructures. The ambient mechanical energy is one of the sources that are abundant and hence different energy harvesting devices and configurations have been reported [1]. Nanogenerators (NGs) using piezoelectric nanowires (NWs) have been developed as a key of technology for converting mechanical energy into electricity. In 2006 Wang et al. introduced the first zinc oxide $(\mathrm{ZnO}) \mathrm{NG}$ based on NWs using atomic force microscopy (AFM) nanoindentation technique and the harvesting piezoelectric potential produced was around 8 $\mathrm{mV}$ with an output power density of $10 \mathrm{pW} / \mathrm{mm}^{2}$ [2]. Then in 2007 Xudong et al. reported a piezoelectric NG based on vertically aligned ZnO NWs arrays which relies on zigzag top electrode. This act like an array of AFM tips that force the NWs to bend in response to external mechanical agitation caused by ultra-sonic wave [3]. Since then various NGs for ultrasonic, vibration, air pressure and body movement energy harvesting have been developed [3, 4]. Never the less until now for scavenging energy from mechanical movement, especially human activity, it has been important to explore wearable and sustainable technologies that function efficiently at low frequencies. Most of the developed NGs based on flexible substrates have shown enhancement in the amount of output power generated under mechanical deformation and can have many applications at low frequency. Beside the relatively high output power performance, flexible substrates do offer multi functionality, e.g. high sensitivity, full flexibility, stretchability, and multi-shape transformability. Therefore, developing piezoelectric energy harvesters with high performance, sensitivity and stretchability is extremely important in order to convert various kinds of mechanical energies available in nature into electricity for the realization of systems operating in self-power mode [5]. The mechanical deformation which causes an output from a NG can be used either as output electric power or as a sensor signal for detecting magnitude and/or the rate of the mechanical deformation. Since no power is applied in the signal detection, sensors based on NGs are called active or self-powered sensors. They have been demonstrated for monitoring heart-pulse, tire pressure, cantilever vibration frequency, ambient wind-velocity detection, transportation, and skin deformation [6].

Among the different piezoelectric materials utilized, one-dimensional $\mathrm{ZnO} \mathrm{NWs}$ have potential for many different technological applications. The attraction to $\mathrm{ZnO}$ nanowires/nanostructures is due to many reasons such as the wide band gap $(3.37 \mathrm{eV})$ combine with relatively large exciton binding energy $(60 \mathrm{meV})$. Zinc oxide also possesses excellent chemical stability, electrical, optical, piezoelectric and pyro-electric properties [7-9]. Among 
the different methods of synthesis of $\mathrm{ZnO}$ NWs, the low temperature hydrothermal method is considered as a promising technique. This is due to the fact that the morphology, structure, and hence properties, can all be controlled by tuning the growth conditions. This is addition to the low cost and ease of fabrication and in addition the low temperature enables the growth on any flexible and foldable substrate [10-11]. However, the morphology of nanomaterials has influence on their properties and the corresponding potential application [12-13]. In particular to piezoelectric nanogenerators, well aligned NWs play an important role in improving the power output [9]. Therefore, to grow nanowires with controlled size, orientation, distribution, and uniformity on any substrates with low cost is of high interest.

Some research on the use of $\mathrm{ZnO}$ nanostructures, including nanowires and nanohemisphere; have been reported for vibration and/or direction detection $[14,15,16]$. A. Yu et al developed a vibration sensor based on $\mathrm{ZnO}$ NWs grown on a cantilever suitable for detecting natural vibrations [14]. While J. Chun et al developed a more complicated configuration based on $\mathrm{ZnO} / \mathrm{PZT}$ hemispheres [15]. In both papers the piezoelectric output voltage observed from an upward or downward bending was having the same polarity but showing different values. The difference between the values obtained from the upward or downward bending was utilized as direction sensor. Another configuration based on four layers of vertically aligned ZnO NWs arrays grown on flexible plastic was reported by B. Saravanakumar et al [16]. Although this configuration could have been utilized for developing direction sensor utilizing the harvested output voltage polarity, such investigation was not carried out. This NG was rather realized as an eco-friendly biomechanical energy harvesting device [16].

In the present work we developed a NG with configuration consisting of $\mathrm{ZnO} N W s$ grown on both sides of a flexible PEDOT: PSS plastic substrate. By measuring the harvested piezoelectric output voltage from the two sides separately, such configuration would be expected to yield different voltage polarities upon bending from the two sides. Since the $\mathrm{ZnO}$ NWs on one side will be compressed while they are stretched on the other side. The morphology and structural properties of the grown $\mathrm{ZnO}$ NWs was investigated by scanning electron microscopy (SEM) and x-ray diffraction (XRD), respectively. The output performance of our double sided NG has been studied under different mechanical deformations by using Keithley 2602A, Digital storage oscilloscope DSO-X 2012A and FET Station 4200-SCS Semiconductor Characterization System. Based on the voltage polarity harvested from the different sides of the NG, we propose this configuration as a sensitive self-powered direction sensor. Moreover the double sided piezoelectric NWs configuration was used to prove the 
possibility of maximizing the output voltage or current when coupling the two sides with different polarity.

\section{Experimental procedure}

\subsection{Growth method}

Zinc nitrate hexahydrate $\left.\left[\mathrm{Zn}(\mathrm{NO})_{3}\right)_{2} 6 \mathrm{H}_{2} \mathrm{O}\right]$, hexamethylenetetramine (HMT) $\left(\mathrm{C}_{6} \mathrm{H}_{12} \mathrm{~N}_{4}\right)$, methanol, ethanol and $\mathrm{KOH}$ were used for synthesis of the $\mathrm{ZnO} \mathrm{NWs}$ in this study. All chemicals were purchased from Sigma-Aldrich and where used without further purification. The growth procedure of well aligned and uniform distributed $\mathrm{ZnO} \mathrm{NWs}$ is described briefly. Firstly, an aqueous solution of $\mathrm{Zn}$ ions is prepared by mixing of $0.05 \mathrm{M}$ of Zinc nitrate hexahydrate in deionized water. Another separate equimolar solution of HMT is homogenous dissolved in deionized. Both solutions were left under stirring for one hour. Secondly, both homogenous solutions were mixed under further stirring at room temperature for another 1 hour. After the growth solution is prepared, a seed solution containing zinc acetate was spun coated on both sides of the substrate at $3000 \mathrm{rpm}$ for $30 \mathrm{~s}$ and then the samples were annealed at $100^{\circ} \mathrm{C}$ for 5 minutes. Finally, the homogenous seed coated substrates were placed vertically in the growth solution and kept in a preheated oven for 5 hours at $70^{\circ} \mathrm{C}$. When the growth duration was completed, the samples were washed with deionized water and dried at for further characterization and device fabrications.

\subsection{Device Fabrication and measurements}

By stacking nanogenerators in series and parallel, higher output piezoelectric power can be achieved [17-18]. Therefore, we grow ZnO NWs on both sides of the substrate and fabricate double layers of $\mathrm{ZnO} \mathrm{NWs}$ on both sides of the substrate in order to increase the output power. The fabrication of the present NG is described as the follows: after successfully growing $\mathrm{ZnO}$ NWs on both sides of the substrates a thin aluminum foil is attached on a top of the $\mathrm{ZnO}$ NWs on both sides and used as ohmic contact. The conducting aluminum sheet was used as the ohmic contact due to it's work function is close to electron affinity of $\mathrm{ZnO}$ and it provides almost ideal ohmic contact with $\mathrm{n}-\mathrm{ZnO}$ [19]. The schematic diagram of the present is shown in Figure 1 (a) and a digital photograph of the NGs under bending is shown in Figure 1 (b). To investigate the energy harvesting process and the energy conversion efficiency of a $\mathrm{ZnO} \mathrm{NWs} \mathrm{NG}$, we measured the output voltage and current signal generated from the device during mechanical deformation. The measurement of the performance of the two sided NG was performed by separately measuring the harvested voltage/polarity and also when connecting the two sides either in series or investigated separately. Two different NGs were fabricated, 
both are having ZnO NWs on both sides, but the first (Type I) is on PEDOT: PSS plastic substrate, while the other (Type II) is on silver coated plastic.

(a)

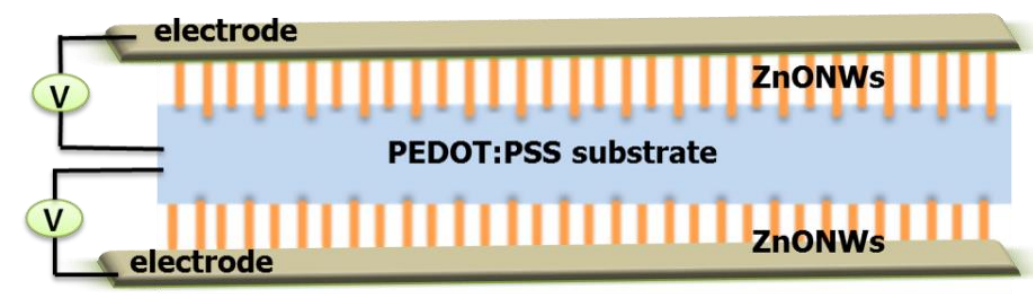

Figure 1 (a) Schematic diagram showing the double sided NG and (b) shows a digital photograph of the NGs under bending.

\section{Results and discussion}

\subsection{Surface morphology and structure characterization}

The surface morphology of the ZnO NWs has been investigated by using SEM. Figure 2a shows a typical SEM image of a grown ZnO sample. From Figure 2 (a), the SEM image of the ZnO NWs indicates a homogeneous and highly dense and well aligned ZnO NWs. The diameter was around $300 \mathrm{~nm}$ and the NWs were almost perpendicular to the surface of the substrate. Figure 2 (b) shows representative XRD patterns of the $\mathrm{ZnO}$ nanowires. All peaks obtained from the grown $\mathrm{ZnO}$ NWs samples were indexed to the hexagonal wurtzite structure of $\mathrm{ZnO}$ and were consistent with the JCPDS No. 36-1451file. Moreover, the high and sharp appearance of the 002 diffraction peak demonstrates that the grown samples have well crystalline nature, and providing evidence that the $\mathrm{ZnO}$ NWs grow along the c-axis $[12,20$ 21].

\subsection{Performance of the double sided NG}

We first investigated the polarity of the harvested voltage from both sides of the $\mathrm{ZnO} \mathrm{NG}$. To perform this measurement the NG is connected as shown in Figure 1a. When the NG sample is bended, the $\mathrm{ZnO}$ NWs on one side will be stretched while they will be compressed on the other side. A piezo-potential will then be produced on both sides. This is illustrated in Figure 3a. As it is well known, stretching and compression will lead to different voltage polarity [6, 15]. According to Alzahrani et al. [22], the bending distortion range of 0 to $360 \mathrm{~nm}$ created on a NW by an atomic force microscope tip induced deflection. The analysis shows that the increasing tip force lead to increase of the piezoelectric polarization and subjecting to the bending angles [22]. Therefore, our experiment presents the relation between the response 
potential and bending angle instead of applied force and we suggest that this nanogenerator can be utilized as a direction sensor rather than a pressure sensor. Hence in our double sided NG and by bending both positive and negative voltage are expected to be produced on the two different sides $[18,19]$. Since the $\mathrm{ZnO}$ NWs were vertically grown onto the substrate, the top of the $\mathrm{ZnO}$ NWs has a positive potential when the NW layer is compressively strained or a negative potential when the NW layer is tensile strained. Therefore, if the decrease of the output voltage was caused by the screening effect from the current leakages and from the impurities according to our assumption, the screening effect should vary with the bending direction. The output voltage and the current were about three times higher when the NW layer was compressively strained than when it was tensile strained, as shown in Figure 3 a [6]. Here the bending is performed by applying a force by hand pressure as shown in Figure $1 \mathrm{~b}$ i.e. this NG operates at low frequency.

(a)

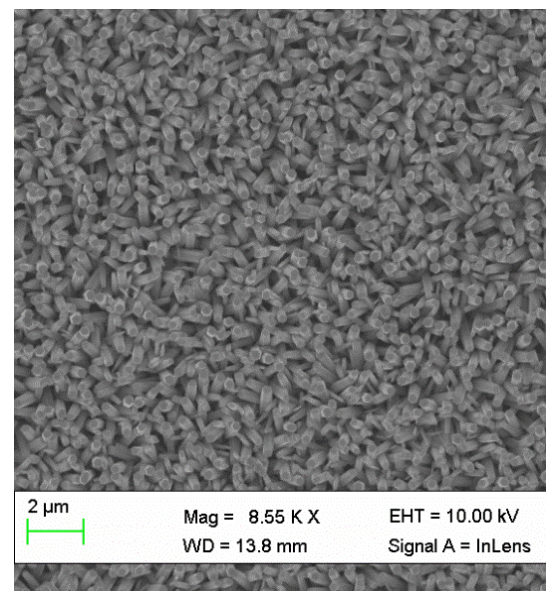

(b)

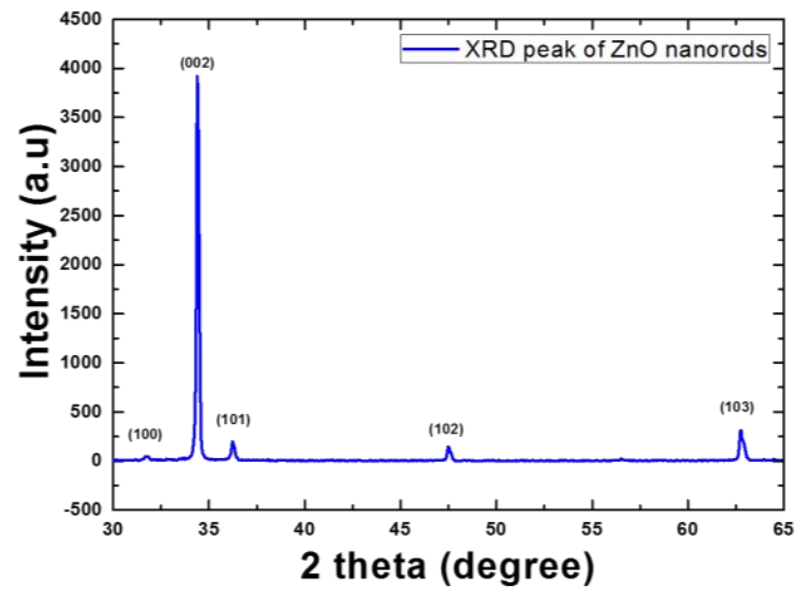

Figure 2 (a) SEM image of the $\mathrm{ZnO}$ nanowires grown on silver coated plastic and (b) XRD patterns of the $\mathrm{ZnO}$ nanowires grown on PEDOT: PSS plastic substrate.

A digital storage oscilloscope DSO-X 2012A was used to measure the output voltage at low frequency when bending the device. Both the top and bottom $\mathrm{ZnO}$ NWs have led to a harvested voltage of $+1 \mathrm{~V}$ and $-1 \mathrm{~V}$, respectively as shown in figure 3 (b). Hence when measuring the harvested voltage from both sides separately, a different voltage polarity is observed as expected. This implies that connecting the two sides of the present NG in series will lead to add up the harvested output. This is consistent with previous published results on connecting many NGs in series [23]. The obtained results are considered as improvement when compared to the harvested output voltage observed from single-sided ZnO NWs NG [6, 14]. Then a FET Station 4200-SCS Semiconductor Characterization System was used for the same NG with 
measurements duration of up to sixty seconds. When applying a downward bending the top side (with bending angle approximately from $30^{\circ}$ to $45^{\circ}$ ) has a compressive strain with positive charge polarity at the top of the NWs, while the bottom side has a tensile strain leading to negative charge polarity at the top of the NWs on the bottom side. So when the NGs is under bending followed by releasing, the response of the output voltage vary between positive and negative polarity for the top and bottom sides, respectively, as shown in figure 3 (c). Also the value and the polarity of the output voltage obtained from the top and bottom NWs are opposite to each other. In addition to that this NG shows stability and reproducibility. The different polarity is due to the different strain on the two sides, while the difference in the value of the observed harvested output voltage is due to the different resistance of the two sides. When bending (figure 3a II) during the bending/releasing we have three different parts. The first part is between 0 to 4 second here we have negative voltage value from the bottom and a positive value from the top side, respectively. The second part is between 5 to 36 second. Here we have higher and lower values in the top-ground and bottom-ground. Finally the third part is between 37 to 60 seconds. Here we observed a negative value in the bottom and a positive value in the top sides, respectively. These observations were stable and reproducible, and it indicates that this double sided NG can be used as a self-powered direction sensor yielding different voltage polarity from the two sides when bended.

Furthermore, in order to investigate the highest energy harvested from the device, we measured the maximum harvested output power from the device at different bending angles ( $2 \theta$ from 0 to $90^{\circ}$ ) as shown in the schematic diagram of figure 4 (a). The measurement was performed for bending (red curves) and releasing (blue curves). This investigation was performed for different connections as shown in the inserts. In general and for all configurations (figure $4 \mathrm{~b}, \mathrm{c}$, and d) the results revealed that the highest harvested output voltage is obtained at a bending angle of about $40^{\circ}$. This is because the smaller the bending angle, the larger the applied strain is on the $\mathrm{ZnO}$ NWs, therefore generating more enhanced output [24]. On other hand, the inordinately high bending angle (above $40^{\circ}$ ) of piezoelectric NWs can lead to the degradation of electromechanical coupling effect and these behaviors will yield low output performance. [25]. It is important to note that the values shown in Figure $4 b, c$, and $d$, are corresponding to the absolute value of the harvested voltage with no consideration of the polarity. In Figure $4 \mathrm{~b}$, and by bending upward (the top NWs are under tensile strain while the bottom NWs are under compressive strain), the maximum harvested output voltage was observed to be $0.06 \mathrm{~V}$. The corresponding value for the bottom NWs (Figure 4c) was observed to be $0.125 \mathrm{~V}$. The difference in value is due to the different resistance between the ground and the bottom and top 
NWs, respectively. Figure 4d shows the harvested output voltage when connecting the top NWs to the bottom NWs. The value obtained for this case was about $0.175 \mathrm{~V}$. Since the geometry of the whole device is actually representing the case of two NGs connected in series the value of the measured output voltage for the case of Figure $4 \mathrm{~d}$ should be expected to be the sum of the values obtained from the separate top and bottom NGs. Indeed the value of $0.175 \mathrm{~V}$ is quite close to the sum which would be $0.185 \mathrm{~V}$. This observation is expected from two NGs connected in series and is also consistent with other published results [23].

(a)

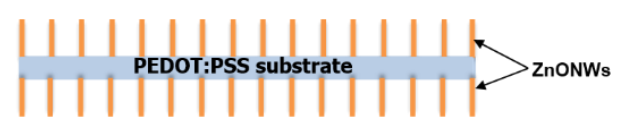

II)

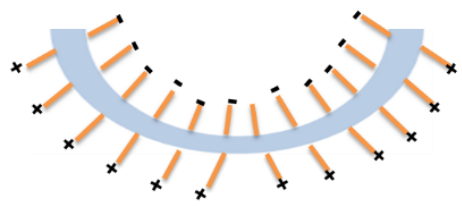

(b)

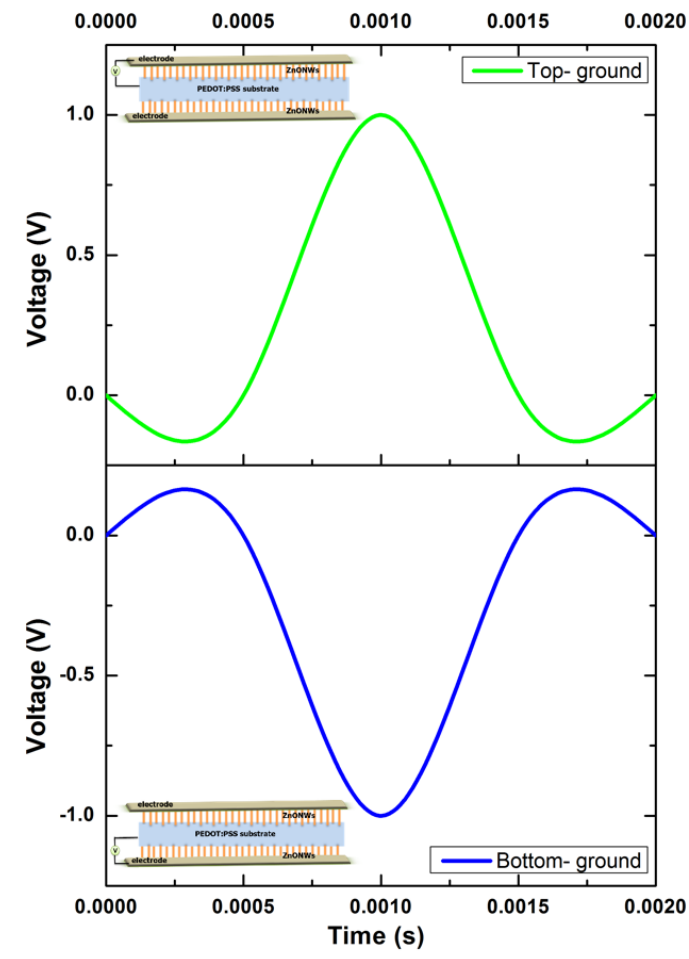

III)

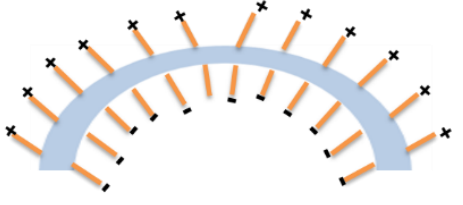

(c)

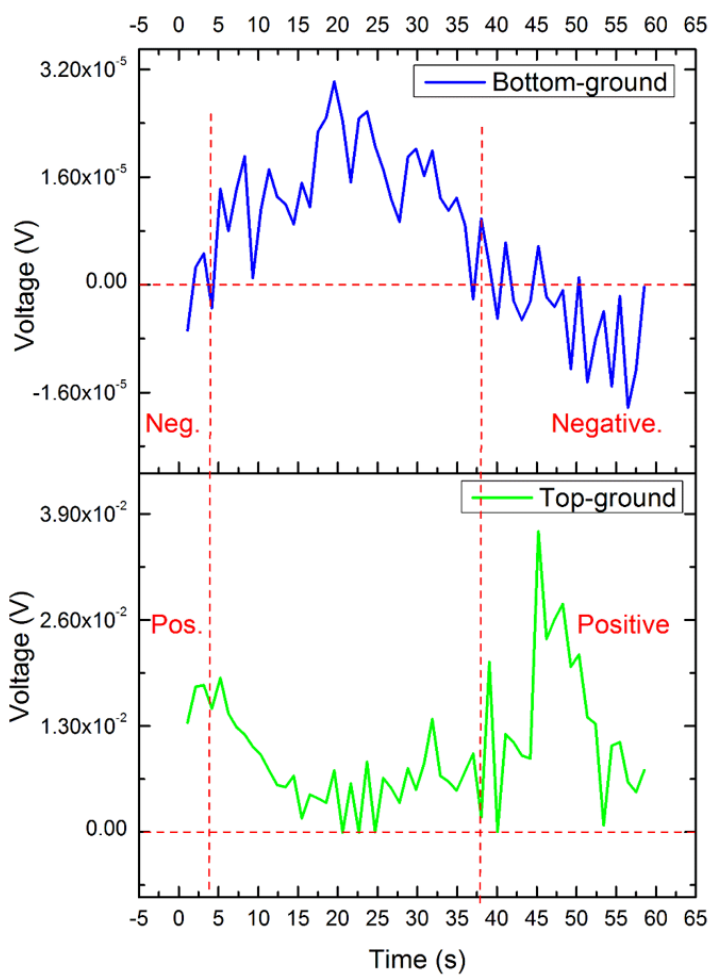

Figure 3 (a) The polarity of the output voltages under bending for case of (I) no bending, (II) downward bending, and (III) upward bending, (b) The measured output voltage for a period of 
millisecond obtained from the top side NWs (green) and bottom side (blue), the inserts show the corresponding measurement side and (c) The generated output voltage from the both sides during compressing and releasing for a period of about 60 seconds.

(a)

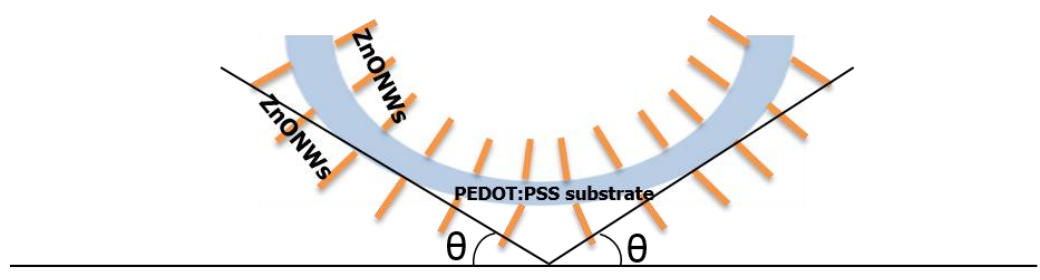

Bending angle $=\mathbf{2 \theta}$

(b)

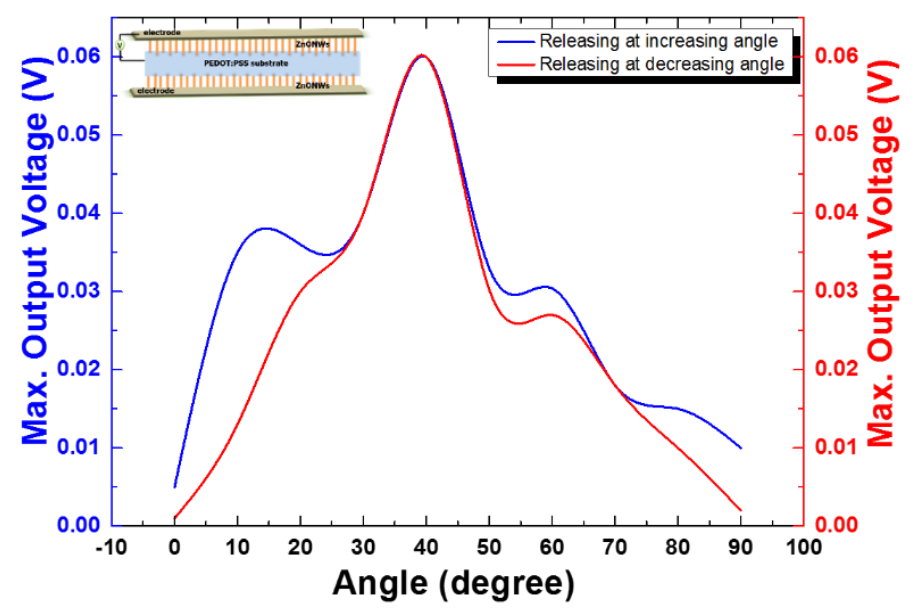

(c)

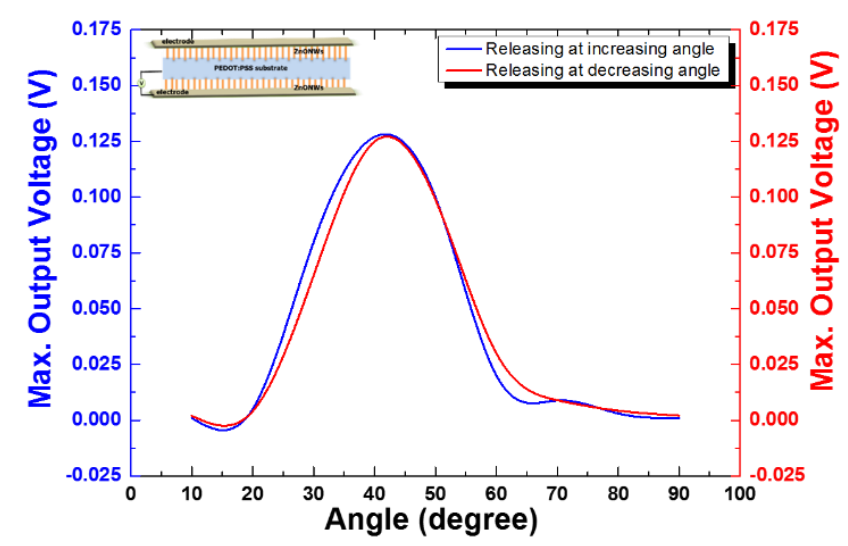

(d) 


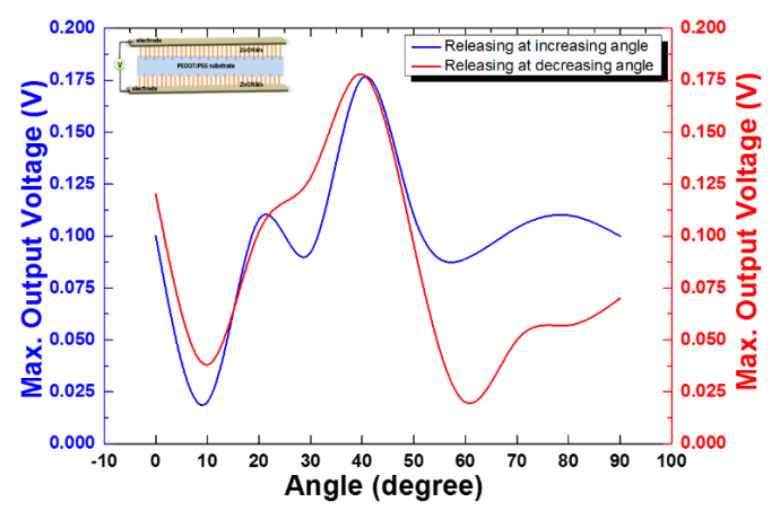

Figure 4: (a) Schematic diagram showing the bending angle measurement configuration. (bd) the maximum output voltage as a function of the bending angle (from 0 to 90 degrees) for the different connections as shown in the inserts of each curve.

Silver coated plastic nanogenerator based on $\mathrm{ZnO}$ NWs grown on both side was fabricated (type II) and used for comparison with the NG based on PEDOT: PSS coated plastic (type I). The study of the output power density for both double sided NGs is shown in figure 5. We measured both the harvested current and voltages for both NGs. Here the bending angle used was chosen to yield the maximum harvested electrical power i.e. $2 \theta$ was around $40^{\circ}$. The current measurements was performed using Keithley 2602A, Keithley's with software that allows automatic data acquisition and make it easy to download recorded data from the instrument. A $100 \mathrm{ohms}$ resistor is connected between the two electrodes and the voltage across the resistor is measured continuously. Then the short circuit current was calculated by using ohm's law. Figure 5 show both instantaneous open circuit voltage and short circuit current from both NGs. Finally, the harvested output power density of the device is estimated according to:

$$
P_{\text {density }}=\frac{P_{\text {out }}}{A}=\frac{I_{\text {out }} \times V_{\text {out }}}{A}=\frac{I^{2} \times R}{A}=\frac{V^{2}}{R \times A}
$$

Where $I_{\text {out }}(\mathrm{A})$ and $V_{\text {out }}(\mathrm{V})$ are instantaneous short circuit current and open circuit voltage, respectively. $A\left(\mathrm{~cm}^{2}\right)$ is the area of the device, $P_{\text {out }}(\mathrm{W})$ is instantaneous output power and $P_{\text {density }}\left(\mathrm{mW} / \mathrm{cm}^{2}\right)$ is instantaneous output power density from the device. Figure $4(\mathrm{a}, \mathrm{b})$ shows the experimental results of type I NG (with PEDOT: PSS). The maximum harvested voltage and current are $2.0 \mathrm{~V}$ and $1.6 \mathrm{~mA}$, respectively with power density amount of 4.44 $\mathrm{mW} / \mathrm{cm}^{2}$. While the maximum generated open circuit voltage, short circuit current and power density of the NG type II were $1.5 \mathrm{~V}, 1.3 \mathrm{~mA}$ and $2.5 \mathrm{~mW} / \mathrm{cm}^{2}$, respectively. The experimental results of type II NG are shown in Figure 4 (c, d). The output results of top-bottom connections shown as a summation of the output between top-ground and bottom-ground connections. 
Many different techniques for the growth of the $\mathrm{ZnO}$ NWs have been used in previous studies of piezoelectric NGs under differ bending. S. Lee et al. generate power density of about $0.011 \mu \mathrm{W} / \mathrm{cm}^{2}$ from a NG based on $\mathrm{ZnO}$ grown on one side of anodic aluminum oxide substrate [6]. J. Chun et al. generate power density of $1.2 \mu \mathrm{W} / \mathrm{cm}^{2}$ from a highly-stretchable composite NG based on stacking three-layers of hemispheres composite in a soft PDMS matrix [15]. Additionally, B. Saravanakumar et al. generate $1.46 \mu \mathrm{W} / \mathrm{cm}^{2}$ power density under folding/releasing a NG based on $\mathrm{ZnO}$ NWs grown on double side PET substrate [16]. Here we used growth of $\mathrm{ZnO}$ NWs on both sides of the flexible substrate and the technique shows an improvement of the harvested output potential compared to other studies using single sided ZnO NWs growth $[19,26]$. One sided NGs have been reported in different studies, the polarity of the harvested voltage depends on the pending direction $[6,14,15]$. Furthermore, from the present double sided NG we have obtained anisotropic voltage polarity from the two sides when bending the NG independently of the direction of bending. Hence, the present double sided NG can act as a self-powered direction sensor to determine the direction of bending.

(a)

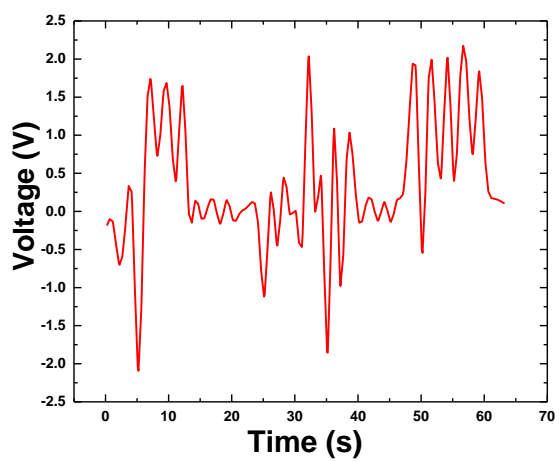

(c)

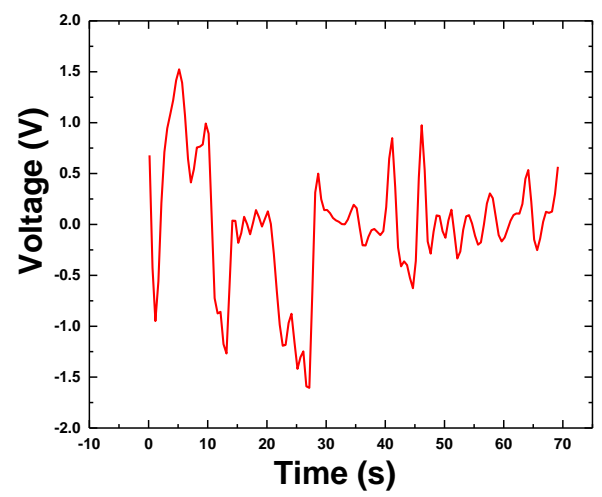

(b)

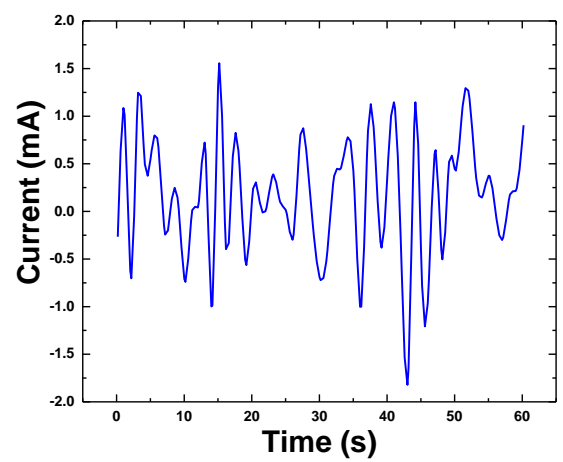

(d)

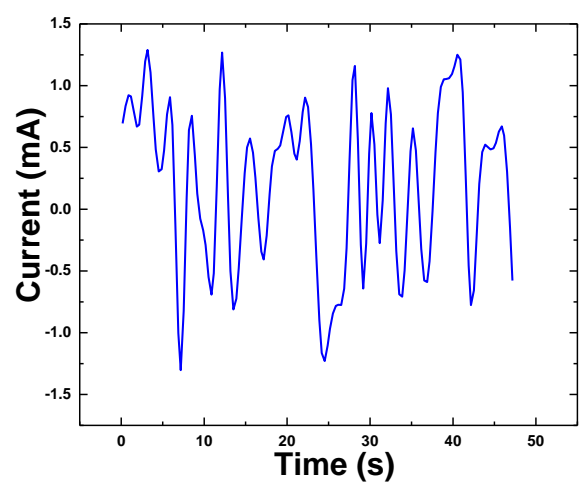

Figure 5: The instantaneous output voltages and current of NG type I (a, b) and NG type II (c, d). 


\section{Conclusion}

We have fabricated a flexible nanogenerator based on $\mathrm{ZnO} \mathrm{NWs}$, hydrothermally grown through a single step on the two sides of a flexible plastic substrate. PEDOT:PSS or silver coated plastic were used, and no pronounced difference in the piezoelectric harvested energy was noted. The energy harvested under different bending angles for the two devices configurations generated $4.44 \mathrm{~mW} / \mathrm{cm}^{2}$ and $2.5 \mathrm{~mW} / \mathrm{cm}^{2}$, from the PEDOT:PSS and silver coated plastic, respectively. Further, the demonstrated results of harvesting mechanical energy from a double sided NG indicated that the output harvested power is doubled compared to the output power harvested form a single side, and is consistent with previously published results of connecting more than one NG in series. The harvested power from each side was found to increase with the bending angel until a maximum bending and upon any further increased bending the harvested powers starts to decrease. This observation was symmetric in nature for bending or releasing procedures. Further, upon bending, each side is either under compressional or tensile strain and this leads to simultaneously generates anisotropic voltage polarity on the two sides. Finally, these results indicate that the present piezoelectric double sided NG can be used as a simple and low cost and stable self-powered direction sensor for many applications, e.g. in safety and security surveillance systems.

\section{Acknowledgements:}

- We would like to thank Gustav Knutsson at ITN, Campus Norrköping, Linköping University, Norrköping, SE-601 4 Norrköping, Sweden, for support during measurements. This research is partially supported by the CeNano grant, and by the Department of Science and Technology, Campus Norrköping, Linköping University, Norrköping, SE-601 4 Norrköping, Sweden. 


\section{References}

1. Yang Y, Zhang H, Lin Z-H, Zhou Y- S, Jing Q, Su Y, Yang J, Chen J, Hu C and Wang ZL 2013 Human Skin Based Triboelectric Nanogenerators for Harvesting Biomechanical Energy and as Self-Powered Active Tactile Sensor System ACS Nano 7 9213-9222.

2. Wang Z-L and Song J 2006 Piezoelectric Nanogenerators Based on Zinc Oxide Nanowire Arrays Science 312, 242-246.

3. Wang X, Song J, Liu J and Wang Z-L 2007 Direct-Current Nanogenerator Driven by Ultrasonic Waves Science 316 102-105.

4. Wang Z-L 2008 Towards Self Powered Nanosystems From nanogenerator to nanopiezotronics Adv. Funct. Mater. 18 3553-3567.

5. Lee J-H, Lee K-Y, Kumar B, Tien N-T, Lee N-E and Kim S-W 2013 Highly sensitive stretchable transparent piezoelectric nanogenerator Energy Environ. Sci. 6 169-175.

6. Lee S, Hinchet R , Lee Y, Yang Y, Lin Z-H , Ardila G, Montès L, Mouis M and Wang ZL 2014 Ultrathin Nanogenerators as Self-Powered-Active Skin Sensors for Tracking Eye Ball Motion Adv. Funct. Mater. 24 1163-1168.

7. Willander M, Israr M-Q, Sadaf J-R and Nur O 2012 Progress on one-dimensional zinc oxide nanomaterials based photonic devices Nanophotonics 1 99-115.

8. Wang Z-L $2009 \mathrm{ZnO}$ nanowire and nanobelt platform for nanotechnology ACSNANO 2 1987-1992.

9. Wang Z-L 2008 One-Dimensional Nanostructures of Zinc Oxide: A New Nanomaterial Family for Nanotechnology Materials Science and Engineering R 64 33-71.

10. Amin G, Asif M-H, Zainelabdin A, Zaman S, Nur O and Willander M 2011 Influence of $\mathrm{pH}$, Precursor Concentration Growth Time, and Temperature on the Morphology of ZnO Nanostructures Grown by Hydrothermal Method Journal of Nanomaterials Article ID 269692, 9 pages.

11. Xu S and Wang Z-L 2011 One-Dimensional ZnO Nanostructures- Solution Growth and Functional Properties Nano Res. 4(11) 1013-1098.

12. Wang S-F, Tseng T-Y, Wang Y-R, Wang C-Y, Lu H-C and Shih W-L 2008 Effects of Preparation Conditions on the Growth of $\mathrm{ZnO}$ Nanorod Arrays Using Aqueous Solution Method Int. J. Appl. Ceram. Technol. 5 (5) 419-429.

13. Sun C and Xue D 2013 Tailoring Anisotropic Morphology at the Nano regime Surface Bonding Motif Determines the Morphology Transformation of $\mathrm{ZnO}$ Nanostructures J. Phys. Chem. C 117 5505-5511.

14. Yu A, Jiang P, Wang Z-L 2012 Nanogenerator as self-powered vibration sensor, Nano Energy doi: 10.1016/ j.nanoen.2011.12.006.

15. Chun J, Kang N-R, Kim J-Y, Noh M-S, Kang C-Y, Choi D, Kim S-W, Wang Z-L and Baik J-M 2014 Highly anisotropic power generation in piezoelectric hemispheres composed stretchable composite film for self-powered motion sensor, Nano Energy (2014), http://dx.doi.org/10.1016/j.nanoen.2014.10.010.

16. Saravanakumar B, Mohan R, Thiyagarajan K and Kim S-J 2013 Fabrication of a ZnO nanogenerator for eco-friendly biomechanical energy harvesting RSC Adv. 3 1664616656.

17. Yang R-S, Qin Y, li C, Dai L-M and Wang Z-L 2009 Characteristics of output voltage and current of integrated nanogenerators Appl. Phys. Letts. 94022905.

18. Yang R-S, Qin Y, Dai L-M and Wang Z-L 2009 Power generation with laterally packaged piezoelectric fine wires Nature Nanotechnology 4 34-39.

19. Lin H-I, Wuu D-S, Shen K-C and Horng R-H 2013 Fabrication of an Ultra-Flexible ZnO Nanogenerator for Harvesting Energy from Respiration ECS Journal of Solid State Science and Technology 2 (9) 400-404.

20. Zhang Y, Ram M-K, Stefanakos E-K and Goswami D-Y 2012 Synthesis, Characterization, and Applications of ZnO Nanowires Journal of Nanomaterials Article ID 624520. 
21. Willander M, Nur O, Zhao Q-X, Yang L-L, Lorenz M, Cao B-Q, Perez J-Z, Czekalla C, Zimmermann G, Grundmann M, Bakin A, Behrends A, Al-Suleiman M, El-Shaer A, Mofor A-C, Postels B, Waag A, Boukos N, Travlos A, Kwack H-S, Guinard J and Dang D-L-S 2009 Zinc oxide nanorod based photonic devices: recent progress in growth, light emitting diodes and lasers Nanotechnology 20332001.

22. Al-Zahrani. H.Y.S, Pal. J, Migliorato. M A. 2013 Non-linear piezoelectricity in wurtzite ZnO semiconductors Nano Energy 2 1214-1217; Al-Zahrani. H.Y.S, Pal. J, Migliorato. M A, Tse. G, Yu. D 2014 Piezoelectric field enhancement in III-V core-shell nanowires Nano Energy http://dx.doi.org/10.1016/j.nanoen.2014.11.046.

23. Cha S-N, Seo J-S, Kim S-M, Kim H-J, Park Y-J, Kim S-W and Kim J-M 2010 SoundDriven Piezoelectric Nanowire-Based NG Adv. Mater. 22 4726-4730.

24. Li X, Lin Z-H, Cheng G, Wen X, Liu Y, Niu S and Wang Z-L 2014 3D Fiber-Based Hybrid Nanogenerator for Energy Harvesting and as a Self-Powered Pressure Sensor NG ACS Nano 8 (10) 10674-10681.

25. Park K-I, Bae S-B, Yang S-H, Lee H-I, Lee K and Lee S-J 2014 Lead-free BaTiO3 nanowires-based flexible composite Nanoscale 68962.

26. Zhu G, Yang R, Wang S and Wang Z-L 2010 Flexible High-Output Nanogenerator Based on Lateral ZnO Nanowire Array Nano Lett. 10 3151-3155. 